\title{
Haptic perception of the horizontal by blind and low-vision individuals
}

\author{
Morton A Heller, Deneen D Brackett, Eric Scroggs, Angela C Allen \\ Department of Psychology, 119 Physical Sciences Building, Eastern Illinois University, Charleston, \\ IL 61920, USA; e-mail: cfmah1@eiu.edu
}

\section{Shavonda Green}

Department of Psychology, Appalachian State University, Boone, NC 28607, USA

Received 10 December 1999, in revised form 26 October 2000

\begin{abstract}
We examined haptic perception of the horizontal in visually impaired people. Blind people (late blind and congenitally blind), persons with very low vision, and blindfolded sighted individuals felt raised-line drawings of jars at four angles. They had to demonstrate their understanding that water remains horizontal, despite jar tilt, by selecting the correct raised-line drawing given four choices. Low-vision subjects, with near perfect scores, performed significantly better than the other groups of subjects. While the late-blind and blindfolded sighted subjects performed slightly better than the congenitally blind participants, the difference between the late-blind and congenitally blind groups was nonsignificant. The performance of the congenitally blind subjects indicates that visual experience is not necessary for the development of an understanding that water level stays horizontal, given container tilt.
\end{abstract}

\section{Introduction}

The purpose of the present study was to examine the understanding of the notion of the horizontal in blind and low-vision adults. If visual experience were necessary for the development of an understanding that water stays horizontal despite tilt of a container, then one would expect an advantage for late-blind or blindfolded sighted adults over congenitally blind people. Performance may suffer if a sensory system is incapable of discovering the physical principle underlying performance in the waterlevel task. One might wonder whether touch has this capability, and, if not, then one would make a negative prediction about the ability of congenitally blind people to perform adequately in the water-level problem (see Revesz 1950). These persons lack visual experience, and must base their understanding of spatial relations upon touch perception.

Piaget studied the development of a child's understanding of horizontal and vertical frames of reference (Piaget and Inhelder 1956) and concluded that sighted children show an understanding of the horizontal and vertical by the end of the concrete operational period. Recent research has shown that many sighted college students often fail to show an understanding that water levels stay horizontal, despite container tilt (Harris et al 1977; Rebelsky 1964; for a review see Voyer et al 1995).

Piaget and Inhelder (1956, page 376) assumed that one's understanding of coordinate frames of reference, the horizontal and vertical, depended upon the ability to ignore transformations of an object and mobile objects within Euclidean space. This understanding of spatial relations presumably extends beyond one's primitive, common-sense understanding that the ground is horizontal and a tree is vertical. Objectively, that is not always the case in the real world. The horizontal and vertical coordinate systems allow an understanding of line, angle and direction in Euclidean space, according to Piaget and Inhelder (1956, see chapter 13 on systems of reference and horizontal-vertical coordinates; also see Millar 1994 for a further discussion of frames of reference in haptics). 
Similarly, according to Millar (1997, page 17):

"To code a configuration spatially as a shape, felt features ...have to be located by reference to each other or to some external or internal frame. The distance, angle and direction of a feature relative to others within a pattern, or its location relative to a background, or to some other spatial frame has to be detected."

Piaget studied reference frames within the context of a variety of concrete problems. The understanding of spatial coordinate systems represents a sophisticated cognitive and perceptual achievement. Performance in the water-level problem is merely one manifestation of cognitive functioning in the face of change, and is dependent upon a number of factors. These factors affecting performance include, but are not limited to, an individual's understanding of the physical principle that water level stays horizontal when a container is tilted. In addition, performance may suffer if an individual has poor spatial or poor haptic perceptual skills. Comprehension of spatial relations within a picture also requires adopting a vantage point, and understanding the spatial relations between the self and the configurations within the depiction. Moreover, it is conceivable that touch is especially susceptible to the distracting effects of container tilt on perception of water angle. Thus, a person may understand the idea that water stays horizontal, but could have some difficulty making concrete judgments about angles using touch. This would make it harder for a congenitally blind person initially to learn about the concept of spatial coordinates (ie the horizontal), and also make it more difficult for someone to respond correctly when subsequently tested. If this analysis is reasonable, one might expect deficiency in the development of spatial understanding in congenitally blind persons.

Hatwell (1985) has studied the development of spatial reasoning in the child, but we have less adequate information about spatial reasoning in visually impaired adults. While there is a relatively large literature available on mobility and map reading, we have less detailed knowledge of the understanding of spatial reference schemes in blind adults (see Millar 1994 for a review).

Robert and her colleagues (Berthiaume et al 1993; Robert and Harel 1996; Robert and Ohlman 1994) reported a great deal of variability when examining the possibility of gender differences in the water-level problem. There are large individual differences in performance, and one occasionally finds a male advantage in the task (eg Robert et al 1994). However, gender differences are not invariably obtained in the water-level task (see Voyer et al 1995), and a male advantage is not found in haptic conditions (Berthiaume et al 1993; Heller et al 1999a; Robert et al 1994). Heller et al (1999a, 1999b) reported minimal gender differences in a haptic task that is very similar to the methodology used in the current study. Consequently, gender was not explicitly selected as a variable of inquiry, and, indeed, cannot be controlled in samples of blind subjects. Complete blindness is so rare that it is impossible to control for gender when selecting visually impaired subjects for research participation.

Low-vision subjects were also included in the study, since they comprise a majority of blind people. However, they have been studied far less than other visually impaired individuals, and it was thought that the presence of minimal visual information deriving from the presence of light perception might facilitate performance in the horizontal water-level problem. Prior research has shown the value of spatial reference information, visual guidance of touch, and the presence of very blurry vision for haptic perception (eg Heller 1982, 1983, 1993, 2000; Millar 1994).

On the assumption that visual experience is necessary for correct solutions of the water-level problem, one would expect deficient performance among congenitally blind individuals. However, if touch were sufficient for the apprehension of the notion that water stays horizontal despite container tilt, congenitally blind people would be expected 
to perform as well as sighted and late-blind subjects. It was not known how the low-vision subjects would perform in this task. However, they were expected to perform at least as well as the late-blind subjects, since most of them had some vision in early childhood.

\section{Experiment 1}

The purpose of experiment 1 was to compare performance of congenitally blind, late-blind, low-vision, and blindfolded sighted subjects in the water-level problem. If visual experience were necessary for learning the concept that water level stays horizontal despite container tilt, congenitally blind subjects would be expected to fail in this task, and perform at a much lower level than the other groups of subjects.

\subsection{Method}

2.1.1 Subjects. The visually impaired subjects were twenty-seven volunteers (twelve males and fifteen females) exhibiting various levels of visual impairment (see table 1). The subjects were recruited from central Illinois, with most of them from Charleston, Danville, Decatur, Champaign, and the Springfield Illinois area. One of the late-blind subjects was recruited in Clinton, Indiana. The nine congenitally blind participants were blind from birth or lost sight well before the end of the first year of life (three males, six females; eight right-handed, one left-handed; mean age $=45.3$ years). The nine late-blind subjects all lost sight after the first year of life (five males, four females; seven righthanded, two left-handed; mean age $=46.3$ years; mean age of loss of sight $=20.8$ years).

In addition, the subjects included nine participants with very low vision (four males, five females; seven right-handed, two left-handed; mean age $=37.3$ years, mean age of onset of visual impairment $=8.1$ years) recruited from the same locations as the blind subjects. A comparison group included nine sighted subjects, recruited on an undergraduate campus (two males, seven females; mean age $=30.1$ years, range $19-45$ years). Low-vision individuals were defined as those with some light, and, at most, some object perception and no more than minimal pattern perception. This group included a wide range in remaining residual vision, with some subjects having the ability to see shadows, some with the ability to see large objects (eg the presence of a large truck), and two subjects with the ability to read large print very slowly with the use of a closed-circuit television (CCTV), great magnification, and very high contrast. None of the subjects was able to see lines on paper, and none could see relatively large objects in front of them. For example, one of the subjects who was able to visually read, given extreme magnification under a CCTV and high contrast, could not see the raised-line drawings under CCTV, and she was a Braille reader. She was also observed walking into another visually impaired person who was directly in front of her. The other person with some minimal form perception was unable to see the raised-line configurations, but could see hand motions. Two elderly subjects with macular degeneration were rejected from the study when it became apparent that they could see the drawings. It was obvious that these subjects had too much residual vision to participate in the study, since, unlike all of the other visually impaired subjects, they did not grope for the drawings and miss them when they initially reached for them. Both of these subjects admitted that they could see the patterns, and one said that she used her peripheral vision to do so. All of the subjects were naïve to the experimental task, and most had no prior experience with tactile pictures.

We should point out that most of the low-vision subjects (all but one) described themselves as 'blind'. The majority of them had no residual pattern perception, and could not see hand motion. One, for example, said that he has no useful vision, but discussion revealed that he could tell when MH's hand was between his eyes and a nearby, strong light source. He had no form or shape perception. Another low-vision subject had no useful pattern perception, and could not tell the direction of a light source. 
Table 1. Characteristics of the blind subjects participating in the Piagetian water-level task, including gender, age, education, age of onset, cause of blindness, and presence of light perception (LP).

\begin{tabular}{|c|c|c|c|c|c|}
\hline Gender & Age/years & Education & Age of onset/years & Cause & LP \\
\hline \multicolumn{6}{|c|}{ Congenitally blind } \\
\hline $\mathrm{F}$ & 27 & HS & 0 & ROP & no \\
\hline $\mathrm{F}$ & 28 & some law & 0 & $\mathrm{RP}$ & yes \\
\hline $\mathrm{F}$ & 36 & JD & 0 & ROP & no \\
\hline M & 45 & MA & 0 & ROP & yes \\
\hline M & 45 & GED & 0 & ROP & no \\
\hline $\mathrm{F}$ & 52 & HS & 0 & $\mathrm{RP}$ & yes \\
\hline $\mathrm{F}$ & 57 & HS & 0 & ROP & no \\
\hline $\mathrm{F}$ & 59 & $\mathrm{PhD}$ & 0 & $\mathrm{ROP}$ & no \\
\hline M & 59 & MA & 0 & congen. glaucoma & no \\
\hline \multicolumn{6}{|c|}{ Late blind } \\
\hline $\mathrm{F}$ & 25 & AS & 16 & virus & yes \\
\hline M & 27 & AS & $\begin{array}{l}\text { LV@ birth, lost } \\
\text { sight@15-16 }\end{array}$ & glaucoma & no \\
\hline M & 55 & $\mathrm{BA}$ & 23 months & retinoblastoma & no \\
\hline $\mathrm{F}$ & 42 & $\mathrm{SC}$ & $\begin{array}{l}\text { LV at birth, at } \\
21 \text { years }\end{array}$ & $\begin{array}{l}\text { topedal retinal } \\
\text { degeneration }\end{array}$ & yes \\
\hline $\mathrm{F}$ & 46 & $\mathrm{BA}$ & 45 & $\mathrm{ROP}$ & no \\
\hline $\mathrm{M}$ & 53 & $\mathrm{PhD}$ & $\begin{array}{l}\text { born with glaucoma; } \\
\text { cataracts, trauma }\end{array}$ & virus & no \\
\hline M & 55 & MA & approx. 2 & retinoblastoma & no \\
\hline M & 57 & HS & 35 & $\begin{array}{l}\text { glaucoma/detached } \\
\text { retina }\end{array}$ & no \\
\hline $\mathrm{F}$ & 57 & $\mathrm{SG}$ & 9 & retinal degeneration & yes \\
\hline \multicolumn{6}{|c|}{ Low vision } \\
\hline $\mathrm{F}$ & 21 & $\mathrm{SC}$ & 6 & ROP & yes \\
\hline $\mathrm{F}$ & 23 & $\mathrm{SG}$ & 23 & blood clots in brain & yes \\
\hline M & 23 & BA & 12 & retinal degeneration & yes \\
\hline $\mathrm{F}$ & 27 & MA & LV at birth & chronic uveitis & yes \\
\hline M & 29 & $\mathrm{SC}$ & 4 & hydrocephalus & yes \\
\hline M & 47 & MA & not known & $\mathrm{RP}$ & yes \\
\hline $\mathrm{F}$ & 51 & HS & birth & $\begin{array}{l}\text { ROP, detached retina, } \\
\text { cataract surgery at } 41\end{array}$ & yes \\
\hline $\mathrm{F}$ & 68 & GED & 20 & macular degeneration & yes \\
\hline M & 47 & AS & 8 & $\mathrm{RP}$ & yes \\
\hline
\end{tabular}

Note: F, female; M, male; LV, low vision; ROP, retinopathy of prematurity; RP, retinitis pigmentosa; SC, some college; SG, some graduate school; AS, junior college degree; BA, college degree; MA, Master of Arts degree; GED, high school equivalency diploma; HS, high school diploma; PhD, Doctor of Philosophy degree; JD, law degree.

She said, however, that she could notice a slight darkness in an area where a number of people stood on campus, and this information aided mobility. Another low-vision subject claimed that he had some residual vision and that he could see 'shadows' on the floor. When asked to indicate where they were, he pointed to a place on the floor that did not have a shadow. To err on the side of conservatism, we decided to include this individual in the low-vision group, since he claimed to have useful vision. All of the low-vision subjects were Braille readers, or knew the Braille code, with the exception of one subject who lost much of her sight a few months before participating in the experiment. 


\subsection{Stimuli and apparatus}

Durable raised-line drawings of jars were produced with a Swedish raised-line drawing kit. The Swedish drawing kit produces a tangible line when a ball point pen is drawn over the surface (see Heller 2000; Heller et al 1996a, 1996b; Kennedy 1993, 1997). This drawing kit can be obtained from the Swedish agency for special education (SIH, Laromedel, Tomtebodavagen 11, 17164 Solna, Sweden). A plastic cylindrical Rubbermaid $2.4 \mathrm{~L}$ (17 cm diameter by $14.5 \mathrm{~cm}$ high) container was filled halfway with water and was presented in both an upright and a tilted manner to allow subjects to feel an actual water surface before touching the picture choices. This container had different proportions than the rectangular depiction of a jar in figures 1a-1c. Nonetheless, the sides of a cylinder are parallel straight lines when viewed from the side, both in the real container and in the raised-line drawing of a jar. A side-view drawing of a cylindrical solid is a rectangle, when the cylinder is viewed at eye-height and at a distance.

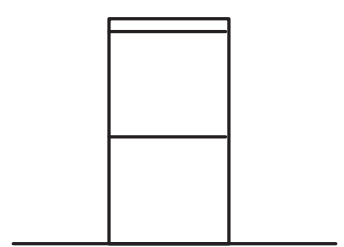

(a)

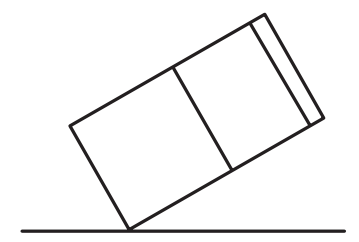

(c)

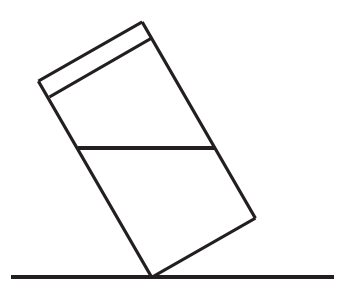

(b)

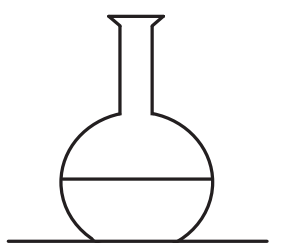

(d)
Figure 1. (a) Standard representation of the jar with $0^{\circ}$ tilt. (b) Representation of water in the jar at $-30^{\circ}$ tilt. (c) Representation of an incorrect choice picture of water in the jar tilted at $60^{\circ}$. (d) Standard representation of the rounded flask with $0^{\circ}$ tilt

A standard drawing (see figure 1a) showed the jar half-full of water, at $0^{\circ}$. The jar was drawn as a rectangle $(7.1 \mathrm{~cm}$ tall by $3.5 \mathrm{~cm}$ wide) on a $10-\mathrm{cm}$ long horizontal line that depicted the table surface. The jar was drawn tilted at four different angles: $-60^{\circ},-30^{\circ}, 30^{\circ}$, and $60^{\circ}$. Jars at negative angles leaned to the left of the vertical; ones at positive angles leaned to the right. Each multiple-choice trial included raised-line drawings of four jars, all at the same angle. One jar had a tangible horizontal line which correctly represented the way the water would settle in the real world, with the surface parallel to the tabletop (see figure 1b). The other three drawings were incorrect representations of the water level in various slanted positions; one erroneous choice was generally parallel to the lid, and the others were $30^{\circ}$ from the correct choice (see figure 1c). The raised-line drawings were mounted on matte board and placed on the tabletop at the subjects' body midline.

\subsection{Design and procedure}

A between - within design was used, with visual status (congenitally blind, late blind, low vision, blindfolded sighted) as the between-subjects variable and angle of the jar $\left(-60^{\circ},-30^{\circ}, 30^{\circ}, 60^{\circ}\right)$ serving as the within-subjects variable. Subjects were not given feedback on performance while executing the task.

Before feeling the sets of pictures, subjects were first asked to feel the surface of the water in the Rubbermaid container, in order to provide a naturalistic experience with water-surface conditions in a tilted container. The subject felt the water surface with free exploration using one or two hands. The experimenter tilted the container $60^{\circ}$ 
(to the right and left) and asked the subject to feel the water in these positions. While the container was tilted and stationary, the subject was told to think about the surface of the water as he/she felt it. The purpose of this procedure was to give subjects an opportunity to discover the physical principle at work in the water-level problem, prior to testing. Of course, no additional verbal feedback was given. This procedure was a modification of one originally used by Piaget and Inhelder (1956). They asked children to first anticipate the effect of container tilt on water level. Subsequently, they sought to determine if children would discover the principle that water level stays horizontal by observation of the effects of motion on the water level. Personal observation of sighted adults (MAH) revealed that some people claimed that water level was actually slanted (and not level; that is, not horizontal) when they looked at a tilted jar that was halffull of water. Obviously, the blind and visually impaired subjects in experiment 1 could not see the water, and so they were asked to feel it at the beginning of the experiment.

Subjects were then presented with the raised-line standard representation of the jar. The subjects were instructed to explore the standard and then asked if they could name the stimulus. None succeeded, but some participants said that the picture seemed like a "window or a hat". Subjects were then told that the picture was a side view of a jar half-full of water. As they felt the lines, they were guided through their exploration to ensure they clearly understood what the component lines represented. The subjects were told that they would be feeling four picture choices of jars that were all tilted at the same angle, but the line representing the water surface would be different for each picture choice. One of the lines represented the way the motionless water would be in the real world if the jar had been superglued in place, remaining still for about $5 \mathrm{~min}$. Subjects were also told that only one of the four choices would be correct, and the other pictures would be incorrect.

Subsequently, as the pictures were presented, subjects were allowed to freely explore them using either one or two hands as they wished, but they were instructed to feel all four choices before making a decision about which one was correct. Subjects were given two random sequences of presentation, with each random sequence containing the 4 possible degrees of jar tilt $\left(-60^{\circ},-30^{\circ}, 30^{\circ}, 60^{\circ}\right)$, for a total of 8 trials. Presentation of the sequences was balanced, so that half of the subjects began with the first sequence, and then they were presented with the second; the rest of the subjects had the reverse order.

\subsection{Results and discussion}

Table 2 illustrates mean number correct overall and by angle for the haptic Piagetian horizontality task. An analysis of variance (ANOVA) was performed on the data obtained from the visually impaired and sighted subjects (congenitally blind, late blind, low vision, blindfolded sighted) on the number of correct judgments. The task was analyzed as a 4 (visual status) $\times 4$ (angle) ANOVA. The results indicated that the main effect of visual status was significant $\left(F_{3,32}=6.25, p<0.01\right)$. However, the main effect of angle failed to reach significance $\left(F_{3,96}=2.2, p>0.091\right)$, as did the angle by visual status interaction $\left(F_{9,96}=1.53, p=0.15\right)$.

Visual experience is clearly not critical for acquiring an understanding of the horizontal, since the congenitally blind subjects performed at a similar level to the blindfolded sighted and late-blind subjects. A Newman-Keuls test on mean number correct showed that the low-vision subjects performed significantly better than all of the other subjects $(p<0.05)$, but the comparisons between the means for the other groups failed to reach significance ( $p>0.05$ for all comparisons).

One reviewer suggested that it would be appropriate to conduct one-tailed $t$-tests on the comparisons between the congenitally blind and other subjects, since the congenitally blind subjects were predicted to perform at a lower level. The comparison 
Table 2. Mean number correct (with standard deviation in parentheses) in Piagetian horizontality task.

\begin{tabular}{llllll}
\hline Subjects & \multicolumn{2}{l}{ Angle of jar/ } & \multirow{2}{*}{ Overall } \\
\cline { 2 - 4 } & -60 & -30 & 30 & 60 & \\
\hline Congenitally blind & $1.1(0.9)$ & $0.7(0.9)$ & $0.8(0.8)$ & $1.0(1.0)$ & $3.6(2.4)$ \\
Late blind & $1.3(0.9)$ & $0.9(1.1)$ & $1.0(1.0)$ & $1.4(0.9)$ & $4.7(3.3)$ \\
Low vision & $2.0(0.0)$ & $2.0(0.0)$ & $1.9(0.3)$ & $1.9(0.3)$ & $7.8(0.7)$ \\
Sighted & $1.0(0.7)$ & $1.6(0.5)$ & $1.0(0.5)$ & $1.7(0.5)$ & $5.2(1.1)$
\end{tabular}

Note: The maximum possible number of judgments per angle correct $=2$; the maximum possible overall number correct $=8$.

between the congenitally blind and late-blind subjects was nonsignificant $(t<1)$. However, one-tailed $t$-tests showed that the congenitally blind subjects performed significantly lower than the low-vision subjects $\left(t_{16}=5.2, p<0.01\right)$ and the blindfolded sighted subjects $\left(t_{16}=1.93, p<0.05\right)$. Vision is not essential for the solution of spatial problems, since the comparison between the congenitally blind and late-blind subjects was nonsignificant.

It should be noted that the low-vision subjects (at about $97 \%$ correct) performed much better than all of the other subjects. Why did the low-vision subjects perform so well in this haptic task? It is suggested that there are probably multiple causes for the advantage of the low-vision participants. It is not prior visual experience, per se, that is critical, since the late-blind subjects performed comparably to the congenitally blind subjects in the present experiment, and the blindfolded sighted subjects in this and in earlier research (Heller et al 1999a).

One possibility is that some of the subjects in the low-vision group could see their hand movements, and this could facilitate spatial cognition and pattern perception in touch (Heller 1982, 1993). However, it should be noted that only three of the subjects in this group reported that they could see hand movements, and the others could not. Another possibility is that low vision helps subjects maintain postural stability, and this could help the subjects. One subject said that vision helped him maintain an upright posture, and perhaps this could aid him in processing spatial reference information. Whatever the explanation, the low-vision subjects had haptic skills that were sufficient for solution of the haptic water-level problem.

\section{Experiment 2}

Perhaps the low-vision subjects were better able to ignore the distracting effects of the tangible surround. Experiment 2 was designed to test the possibility that an important component of the difficulty in the haptic version of the water-level problem involves the configuration of the jar. Experiment 2 was designed to study the effect of jar configuration, by comparing performance of blindfolded sighted subjects tested with rectangular jars with a similar task, but with rounded (flat-bottom) flasks. If jar configuration distracts subjects using touch, then one might expect better performance with rounded flasks (see figure 1d). This experiment eliminated knowledge of physical principles as a variable, since subjects were given prior knowledge that water level stays horizontal at the beginning of the experiment.

\subsection{Method}

3.1.1 Subjects. There were two groups of blindfolded sighted subjects (fifty-six, twentyeight per group, with equal numbers of males and females in each group). 


\subsection{Stimuli and apparatus}

These were similar to experiment 1 , except that one set of stimuli involved rounded flasks (see figure 1d).

\subsection{Design and procedure}

Subjects were told to find the horizontal line when exposed to a multiple-choice Piaget horizontality task, as in experiment 1 . One group of subjects had rectangular jars, and the second had rounded flasks. Both groups were told that the water level stays horizontal, and flat like the tabletop, prior to feeling any of the stimuli.

\subsection{Results and discussion}

Performance was significantly superior for the group given the rounded flasks (mean $=7.4$ out of 8 trials) than for the group with the raised-line pictures of the jars $($ mean $=4.8)\left(F_{1,54}=15.6, p=0.000\right)$. These results indicate that a substantial part of the task's difficulty probably derives from the distracting effects of the tangible surround, as in the rod-and-frame task (see Robert and Ohlman 1994). Note that the subjects participating in the rectangular-jar condition did not benefit from the verbal instructions about the horizontal, since their scores were slightly lower than those of the blindfolded sighted group (mean $=5.2$ correct) in experiment 1 reported here. It is also noteworthy that the blindfolded sighted subjects (in experiment 1) were given haptic, but not verbal, information about the results of tilt, since they were able to feel the water surface before the start of the experiment. These data are consistent with the idea that low-vision subjects in experiment 1 were apparently able to ignore the distracting effects of the rectangular-jar surround, since their performance was nearly perfect. Of course these conclusions must be considered speculative, and tempered by the possibility that the low-vision subjects were exceptional. This explanation is taken up more fully at a later point in this report.

\section{General discussion}

Visual experience is clearly not necessary for an understanding that water level stays horizontal given container tilt. The performance of the congenitally blind subjects was somewhat lower than that of the late-blind subjects, but not significantly worse. There were large individual differences among the early-blind subjects, with some showing high levels of performance. These results further support the notion that haptics may often substitute for a lack of vision or prior visual experience (see Millar 1994). However, this comment does not diminish the striking and robust advantage of the low-vision subjects in the present study.

The following comments on the advantage of the low-vision subjects should be identified as speculative. The samples of subjects in the experiments reported here are small, and we know that there are large individual differences in performance in the water-level task and in haptics. Thus, it is conceivable that the low-vision subjects are simply exceptional subjects. Seven of the nine low-vision subjects were either rehabilitation counselors for blind people, or they were directors of support groups for visually impaired people. People with poor spatial skills are unlikely to become rehabilitation educators or organizers of support groups.

Perhaps the low-vision subjects developed superior haptic perceptual skills, and also did not suffer the distracting effects of visual pattern information during the process of learning to make use of touch. Some of these individuals may have learned to effectively ignore distracting visual input. Visual pattern information can sometimes distract subjects when they are trying to attend to tactile pattern information or learn tactile skills (see Heller 1992, 1993; Heller et al 1999b). The present analysis assumes that vision can serve a dual function in its relation to touch; that is, it can provide pattern information, or it can provide guidance of haptic exploration. An inappropriate reliance 
on vision for pattern perception as vision deteriorates may developmentally delay the acquisition of superior haptic skills. Perhaps the low-vision subjects in the present study learned to make use of remaining residual vision for spatial frame-of-reference information. Note, too, that blurry vision can induce haptic dominance, and the results of this experiment are consistent with earlier reports (Heller 1983).

We should point out that many of the low-vision subjects described themselves as blind, and claimed that they were not able to make much use of visual information. Most of them were Braille readers, and used a long cane for mobility. It was only upon careful, detailed questioning, that we discovered that some of them, for example, made use of light perception to maintain an upright posture. This postural precision can further help an individual use a body-centered framework to guide haptic exploration (see Millar 1994). Another low-vision subject remarked that she could not see forms, contours, or hand movements, but often used minimal brightness differences to coarsely localize extremely large objects (eg a building) in space.

The low-vision subjects actually performed marginally better than a sample of sighted college students using vision, ${ }^{(1)}$ and that speaks well for the potential of haptics. We should also point out that the low-vision subjects lost their sight at an earlier age than the late-blind subjects did. They had more time to forget visual impressions, but also had earlier exposure to education in the use of touch for pattern perception and mobility. Thus, they had the benefits of early experience in education of touch, and also had any advantages that accrued from prior visual experience and from their remaining residual vision. It is possible that this minimal residual vision has positively altered their spatial skills, contributing to the superiority of the low-vision subjects in haptics.

However one interprets the advantage of the low-vision subjects, it is inappropriate to assume that an individual will perform poorly on the water-level problem because of a visual impairment. Low-vision subjects performed excellently. Moreover, the success of the congenitally blind subjects suggests that touch is able to provide information about the horizontal orientation of the water line. One can feel the surface of liquids in a glass with a finger, and one can also feel the water line while one is partially submerged in a lake, or swimming pool, or a bathtub. Clearly, touch provides information that will support understanding of the horizontal spatial coordinate.

Acknowledgements. Preparation of this report and some of the research were supported by NIH ROI EY12040. Faith Heller provided helpful comments on an earlier version of this manuscript. We are grateful to Shana Salik for assistance with data collection and analysis.

(1) Low-vision subjects may have a better understanding of the horizontal than other individuals. Of course, these comments are speculative, and the advantage of the low-vision subjects could be ascribed to exceptionality. To understand just how well they performed, we replicated the experiment using sighted subjects, but with vision of the same drawings as those used in the prior haptic conditions. In addition, blue tarpaulins were hung in an arc around the walls of the room to eliminate visual information about horizontals or verticals. Subjects (nine; four males, five females) were sighted undergraduates recruited from introductory psychology classes. They were allowed to see the actual jar of water at the upright and then tilted $60^{\circ}$ to the left and right. They were told to look at the jar and attend to the water surface, but were not allowed to touch the water. These sighted subjects were given no additional prior information about the solution to the water-level problem, and in all important respects their instructions were identical to those for the haptic conditions for the visually impaired subjects. The only alteration in instructions derived from the change in modality to vision. Visual performance for the sighted group (mean $=6.7$ correct) was lower than that for the low-vision group using haptics (mean $=7.8$ correct), and the difference between the means was marginally significant ( $t_{16}=2.03, p=0.059$, two-tailed). 


\section{References}

Berthiaume F, Robert M, St-Onge R, Pelletier J, 1993 "Absence of a gender difference in a haptic version of the water-level task" Bulletin of the Psychonomic Society 31 57-60

Harris L J, Hanley C, Best C T, 1977 "Conservation of horizontality: Sex differences in sixth graders and college students", in Readings in Child Development and Relationships Eds R C Smart, M S Smart (London: Macmillan) pp 375-387

Hatwell Y, 1985 Piagetian Reasoning and the Blind (New York: American Foundation for the Blind)

Heller M A, 1982 "Visual and tactual texture perception: Intersensory cooperation" Perception \& Psychophysics $31339-344$

Heller M A, 1983 "Haptic dominance in form perception with blurred vision" Perception 12 $607-613$

Heller M A, 1992 “ “Haptic dominance' in form perception: Vision versus proprioception” Perception $21655-660$

Heller M A, 1993 "Influence of visual guidance on Braille recognition: Low lighting also helps touch" Perception \& Psychophysics 54 675-681

Heller M A, 2000 Touch Representation and Blindness (Oxford: Oxford University Press)

Heller M A, Calcaterra J A, Burson L L, Tyler L A, 1996a "Tactual picture identification by blind and sighted people: Effects of providing categorical information" Perception \& Psychophysics $58310-323$

Heller M A, Calcaterra J A, Tyler L A, Burson L L, 1996b "Production and interpretation of perspective drawings by blind and sighted people" Perception 25321 - 334

Heller M A, Calcaterra J A, Green S L, Barnette S L, 1999a "Perception of the horizontal and vertical in tangible displays: minimal gender differences" Perception 28 387-394

Heller M A, Calcaterra J A, Green S L, Brown L, 1999b "Intersensory conflict between vision and touch: The response modality dominates when precise attention-riveting judgments are required" Perception \& Psychophysics 61 1384-1398

Kennedy J M, 1993 Drawing and the Blind (New Haven, CT: Yale University Press)

Kennedy J M, 1997 "How the blind draw" Scientific American 276(1) 76-81

Millar S, 1994 Understanding and Representing Space: Theory and Evidence from Studies with Blind and Sighted Children (Oxford: Oxford University Press)

Millar S, 1997 Reading by Touch (London: Routledge)

Piaget J, Inhelder B, 1956 The Child's Conception of Space (New York: W W Norton)

Rebelsky F, 1964 "Adults perception of the horizontal" Perceptual \& Motor Skills 19 371-374

Revesz G, 1950 The Psychology and Art of the Blind (London: Longmans Green)

Robert M, Harel F, 1996 "The gender difference in orienting liquid surfaces and plumb-lines: Its robustness, its correlates, and the associated knowledge of simple physics" Canadian Journal of Experimental Psychology 50280 - 314

Robert M, Ohlman T, 1994 "Water-level representation by men and women as a function of rod-and-frame test proficiency and visual and postural information" Perception 231321 - 1333

Robert M, Pelletier J, St-Onge R, Berthiaume F, 1994 "Women's deficiency in water-level representation: Present in visual conditions yet absent in haptic contexts" Acta Psychologica 87 19-32

Voyer D, Voyer S, Bryden M P, 1995 "Magnitude of sex differences in spatial abilities: A metaanalysis and consideration of critical variables" Psychological Bulletin 117 250-270 